\title{
ZONEAMENTO AGROCLIMÁTICO DA CULTURA DA MANGUEIRA NO ESTADO DO PIAUÍ
}

\author{
GILSONLAGES FORTES PORTELA², MILCIADES GADELHADE LIMA ${ }^{3}$, LUIZEVALDO DE MOURAPADUA $^{4}$, \\ FRANCISCO DE ASSIS SINIMBU NETO ${ }^{5}$, ANTÔNIO BALDO GERALDO MARTINS ${ }^{6}$
}

RESUMO - O presente trabalho teve como objetivo realizar o zoneamento agroclimático da cultura da mangueira no Estado do Piauí. Foram utilizados dados médios anuais de precipitação e temperatura do ar, de 15 municípios do Piauí, representativos das principais regiões do Estado para a elaboração do balanço hídrico e a obtenção do Índice de Umidade (Im). Foram adotadas as seguintes faixas de Índice de Umidade (Im): - Aptidão plena A, Im <-40; - Aptidão plena $\mathbf{B},-40<\operatorname{Im}<-20$. O critério utilizado na divisão do Estado, em zonas térmicas, baseou-se nas normais de temperaturas médias do mês mais quente (Tq) para cada local. Assim sendo, as faixas $\mathbf{A}$ e $\mathbf{B}$, com aptidão plena, do ponto de vista hídrico, foram subdivididas em duas subzonas: Aptidão plena $\mathbf{A}_{1}:\left(\mathbf{T q}<29^{\circ} \mathrm{C}\right)$, Aptidão plena $\mathbf{A}_{2}$ : $\left(29^{\circ} \mathbf{C}<\mathbf{T q}<31^{\circ} \mathrm{C}\right)$ e Aptidão plena $\mathbf{B 1}$ : $\left(\mathbf{T q}<29^{\circ} \mathrm{C}\right)$, Aptidão plena $\mathbf{B}_{2}:\left(29^{\circ} \mathrm{C}<\mathrm{Tq}<31^{\circ} \mathrm{C}\right)$, para atender ao critério térmico. Com base nas cartas de Tq e Im, foram definidos os limites para as diferentes zonas de aptidão climáticas para o cultivo de manga. À luz dos critérios utilizados e com base na literatura consultada, o Estado do Piauí não apresenta nenhuma restrição climática para o cultivo comercial da mangueira.

Termos para indexação: Aptidão climática, Mangifera indica, índice de umidade.

\section{AGROCLIMATIC ZONING OF MANGO CROP IN THE STATE OF PIAUI}

ABSTRAT - The present work had as objective to make the agroclimatic zoning of the mango crop in the State of Piauí. The average annual data of precipitation and temperature of air, of 15 cities of Piauí, were utilized representing the main regions of the State for the elaboration of the water balance and attainment of the moisture index $(\mathrm{Im})$. The following bands of moisture Index had been adopted (Im): Full aptitude A, Im <- 40; Full aptitude B,- $40<\operatorname{Im}<-20$. The criterion utilized in the division of the state in thermal areas was based on the normal temperatures measured of the hottest month $(\mathrm{Tq})$ for each place. Then, the bands $\mathrm{A}$ and $\mathrm{B}$ with full aptitude of the hydric point of view had been subdivided in two subareas: Full aptitude $\mathrm{A}_{1}$ : $\left(\mathbf{T q}<29^{\circ} \mathrm{C}\right)$, Full aptitude $\mathbf{A}_{2}:\left(29^{\circ} \mathrm{C}<\mathbf{T q}<31^{\circ} \mathrm{C}\right)$ and Full aptitude B1: $\left(\mathbf{T q}<29^{\circ} \mathrm{C}\right)$, Full aptitude $\mathbf{B}_{2}:\left(29^{\circ} \mathrm{C}<\mathrm{Tq}<31^{\circ} \mathrm{C}\right)$. Based on the letters of Tq and $\mathrm{Im}$, the limits for different climatic areas of aptitude for the mango crop were defined. Based on the used criterions and on the consulted literature, the state of Piaui does not present none climate restriction for the commercial mango cropping.

Index terms - Climate aptitude, Mangifera indica, moisture index.

\section{INTRODUÇÃO}

O Brasil possui condições ecológicas favoráveis à exploração comercial de fruteiras. Com uma produção de 320 milhões de toneladas, o País é responsável por cerca de $10 \%$ da produção mundial. Entre as culturas cultivadas, a manga (Mangifera indica L.) destaca-se. Essa fruteira é explorada praticamente em todas as regiões do País. No Nordeste, encontra condições ambientais bastante adequadas, principalmente altas temperaturas e baixa umidade, fazendo com que os frutos da região apresentem sabor e cor excelentes, características fundamentais para o mercado consumidor (Medeiros et al.,2005). Os maiores produtores são os Estados de São Paulo, Pernambuco, Bahia e Minas Gerais. O Estado do Piauí apresenta condições favoráveis à exploração comercial da cultura, sendo um tradicional produtor (Francisco et al., 2003).
O crescimento e o desenvolvimento da planta são influenciados pelo ambiente. $\mathrm{O}$ estudo das respostas da planta às diferentes condições climáticas é de fundamental interesse no estabelecimento do melhor manejo para a cultura (Pinheiro et al., 2002). As mangueiras adaptam-se às mais variadas condições edafoclimáticas tropicais e subtropicais, sendo que as melhores regiões para a produção comercial da cultura são aquelas que apresentam frio e/ou período seco que antecede a floração, solo bastante úmido e temperatura máxima do ar em torno de $30-33^{\circ}$ C durante o desenvolvimento do fruto. A manga pertence ao grupo de plantas tolerantes à seca, mas pode resistir a curtos períodos de cheia (Schaffer \& Andersen, 1994). Temperaturas máximas acima de $35^{\circ}$ causam injúrias nas plantas, já temperaturas de 8 a $10^{\circ} \mathrm{C}$ durante o período frio do ano podem ser consideradas como o limite inferior para o sucesso na produção de manga (Campbell, 1994).

\footnotetext{
1(Trabalho 012-08) Recebido em 31-01-2008. Aceito para publicação em: 13-08-2008.

${ }^{2}$ Eng. Agr., Aluno de Mestrado do Programa de Pós-Graduação em Agronomia, CCA/UFPI, gilsonportela@uol.com.br ${ }^{3}$ Prof. Dr., Eng. Agr., Professor Associado, Universidade Federal do Piauí, CCA, Campus da Socopo, Teresina, Piauí, Gadelha@ufpi.br ${ }^{4}$ Prof. Dr., Eng. Agr., Professor Associado, Universidade Federal do Piauí, CCA, Campus da Socopo, Teresina, Piauí, lempadua@ufpi.br ${ }^{5}$ Eng. Agr.,Aluno de Doutorado do Programa de Pós-Graduação em Agronomia-Produção Vegetal, UNESP-Jaboticabal, f.sinimbu@ig.com.br ${ }^{6}$ Prof. Dr., Eng. Agr., Professor Assistente Doutor da Universidade Estadual Paulista Júlio de Mesquita Filho, Jaboticabal, São Paulo, baldo@ fcav.unesp.br
} 
A cultura exige, para produzir maiores safras e melhores frutos, a existência de uma estação seca bem definida (Brunini \& Alfonsi, 1980). Essa estação é necessária para que a planta entre em dormência temporária, com isto ocorre diminuição do crescimento vegetativo e, como conseqüência, o aumento da produção de flores e frutos. A mangueira cultivada sob baixa precipitação (1.000 a $1.500 \mathrm{~mm}$ ) necessita de irrigação para produzir bem, já em alta precipitação (1.900 a $2.500 \mathrm{~mm}$ ) necessita de um eficiente sistema de drenagem (Castro \& Kluge, 1998).

Considerando a importância da cultura para o Estado do Piauí, objetivou-se elaborar o zoneamento agroclimático da cultura da mangueira para as condições climáticas do Estado.

\section{MATERIAL E MÉTODOS}

Para o presente estudo, foram utilizados dados de precipitação e temperatura do ar de 15 municípios do Piauí, representativos das principais regiões do Estado (Tabela 1). Os dados de precipitação foram obtidos na Gerência de Hidrometeorologia da Secretaria Estadual do Meio Ambiente e Recursos Hídricos. Obteve-se a média mensal de 40 anos (1963/ 2003) de dados. Os valores de temperatura do ar foram estimados de acordo com Lima e Ribeiro (1999) por meio da equação de regressão linear múltipla, a seguir:

$$
\mathrm{E}(\mathrm{Y})=\mathrm{a}_{0}+\mathrm{a}_{1} \mathrm{X}_{1}+\mathrm{a}_{2} \mathrm{X}_{2}+\mathrm{a}_{3} \mathrm{X}_{3} \text {, em que: }
$$

$\mathrm{Y}=$ temperatura mensal do ar (média); $\mathrm{X}_{1}=$ latitude do local (min); $X_{2}=$ altitude do local ( $\left.\mathrm{m}\right) ; \mathrm{X}_{3}=$ longitude do local (min); $a_{0}, a_{1}, a_{2}$ e $a_{3}=$ são os coeficientes da equação de regressão.

Foram utilizados dados médios anuais de precipitação e temperatura do ar, de 15 municípios do Piauí, representativos das principais regiões do Estado para a elaboração do balanço hídrico e a obtenção do índice de umidade anual (Im).

O cálculo do balanço hídrico climatológico foi feito com o software "Balanços Hídricos Climatológicos do Brasil" (Sentelhas et al., 1999), adotando-se o método de Thornthwaite \& Mather (1955) para uma capacidade de água disponível (CAD) de $100 \mathrm{~mm}$, com a evapotranspiração potencial (ETP), sendo estimada pelo método de Thornthwaite (1948).

O índice de umidade anual ( $\mathrm{Im})$, revisado do método de Thornthwaite e Mather (1955), pode ser escrito como: $\mathrm{Im}=[(\mathrm{P}-$ ETP) / ETP] x 100, em que: $\mathrm{Im}=$ índice de umidade anual; $\mathrm{P}=$ $\operatorname{precipitação~anual~}(\mathrm{mm}) ; \mathrm{ETP}=$ evapotranspiração potencial $(\mathrm{mm})$ (Krishnan, 1980).

Com as informações disponíveis na literatura e com os dados dos balanços hídricos, obtiveram-se os índices climáticos limites (Teixeira e Azevedo, 1996) modificados, para atender às condições climáticas do Estado do Piauí para o cultivo de manga, os quais serviram de base para a classificação do grau de aptidão climática das diversas regiões do Estado.

Foram adotadas as seguintes faixas de índice de umidade anual (Im) de Thornthwaite \& Mather (1955): Aptidão plena $\mathbf{A}(\operatorname{Im}<-40)$ e Aptidão plena $\mathbf{B}(-40<\operatorname{Im}<-20)$.

O critério utilizado na divisão do Estado em zonas térmicas, baseou-se nas normais de temperaturas médias do mês mais quente (Tq) para cada local. Assim sendo, as faixas A e B, com aptidão plena, do ponto de vista hídrico, foram subdivididas em duas subzonas: Aptidão plena $\mathbf{A}_{\mathbf{1}}$ : $\left(\mathbf{T q}<29^{\circ} \mathrm{C}\right)$, Aptidão plena $\mathbf{A}_{2}:\left(29^{\circ} \mathrm{C}<\mathbf{T q}<31^{\circ} \mathrm{C}\right)$ e Aptidão plena $\mathbf{B 1}:\left(\mathbf{T q}<29^{\circ} \mathrm{C}\right)$, Aptidão plena $\mathbf{B}_{2}:\left(29^{\circ} \mathrm{C}<\mathrm{Tq}<31^{\circ} \mathrm{C}\right)$, para atender ao critério térmico.

Com base nas cartas de Im e Tq, foram definidos os limites para as diferentes zonas de aptidão climáticas para o cultivo de manga.

\section{RESULTADOS E DISCUSSÃO}

A Tabela 2 contém os valores de Tq, P, Im e os resultados do balanço hídrico para 15 municípios representativos das diversas regiões do Estado do Piauí. Os balanços hídricos anuais variaram de -87,6 mm em (Piripiri) a - 1.095,9 mm (Picos). A precipitação média anual variou entre 696,8 mm (São Raimundo Nonato) e 1.584,2 mm (Piripiri), ficando todos os 15 municípios dentro das exigências hídricas para o cultivo da mangueira, segundo Silva (1982).

Segundo a classificação climática de Thornthwaite e Mather (1955), o índice de umidade anual (Im) variou de -5,2 (Piripiri) a -60,8 (Picos), que correspondem aos tipos climáticos subúmido seco e semi-árido. Com esses dados, percebe-se que os municípios localizados em áreas de clima semi-árido apresentam grandes deficiências hídricas, o que favorece o florescimento da cultura.

A Tq (Temperatura média do mês mais quente) variou de $27,3^{\circ} \mathrm{C}$ (Corrente) a $29,5^{\circ} \mathrm{C}$ (Floriano). A cultura da mangueira produz satisfatoriamente numa ampla faixa de temperatura média que vai de $25^{\circ} \mathrm{C}$ a $30^{\circ} \mathrm{C}$, segundo Schaffer \& Andersen, (1994) e Campbell. (1994), mostrando que, para os municípios avaliados, a cultura da mangueira não tem limitação térmica.

Na delimitação das áreas da aptidão climática para a cultura da mangueira (Figura 1), foram consideradas as exigências normais da espécie. Como são muitas as variedades comerciais, algumas mais exigentes, outras menos, a carta deve ser considerada como uma indicação geral. As faixas consideradas de aptidão plena apresentam condições climáticas favoráveis a praticamente todas as variedades.

Pelos dados apresentados, verifica-se que é possível o cultivo da mangueira nas faixas A e B, mesmo com elevada deficiência hídrica na maioria dos meses do ano, conforme os dados do balanço hídrico anual (Tabela 2). Quanto às necessidades térmicas, essas faixas satisfazem plenamente à cultura. As diferenças existentes entre as faixas A e B são quanto ao índice umidade (Im). Os municípios localizados na faixa $\mathrm{A}$, principalmente na Faixa $\mathrm{A}_{1}$, são os mais propícios à instalação de plantios comerciais, pois apresentam elevadas temperaturas, deficiências hídricas elevadas e baixa umidade do ar, sendo poucos os problemas com doenças, fator de grandes prejuízos, além de um período seco maior, o que favorece o florescimento da cultura.

À luz dos critérios utilizados neste estudo, verifica-se que o município de Piripiri, ainda que não pertencesse à aptidão plena, foi deslocado para a faixa $\mathrm{A}_{1}$ por se enquadrar melhor nesse contexto, ou seja, em termos de disponibilidades térmicas 
essa faixa não apresenta restrições. Do ponto de vista hídrico, é viável o cultivo de manga em meses que apresentam excedentes hídricos elevados, com a utilização de sistema de drenagem.

Para uma análise mais criteriosa, devem-se considerar não apenas os valores de temperatura do ar e precipitação, mas também a combinação dos diversos parâmetros meteorológicos, tais como umidade relativa do ar, ventos, luminosidade, altitude e latitude.

TABELA 1-Coordenadas geográficas dos municípios representativos das principais regiões do Estado do Piauí.

\begin{tabular}{|c|c|c|c|}
\hline Municípios & $\begin{array}{l}\text { Latitude } \\
\end{array}$ & Longitude & Altitude (m) \\
\hline Parnaíba & $02^{\circ} 55^{\prime} \mathrm{S}$ & $41^{\circ} 47^{\prime} \mathrm{W}$ & 15 \\
\hline Piripiri & $04^{\circ} 17^{\prime} \mathrm{S}$ & $41^{\circ} 47^{\prime} \mathrm{W}$ & 160 \\
\hline Campo Maior & $04^{\circ} 49^{\prime} \mathrm{S}$ & $41^{\circ} 11^{\prime} \mathrm{W}$ & 125 \\
\hline Teresina & $05^{\circ} 05^{\prime} \mathrm{S}$ & $42^{\circ} 49^{\prime} \mathrm{W}$ & 72 \\
\hline São Pedro do PI & $05^{\circ} 56^{\prime} \mathrm{S}$ & $42^{\circ} 44^{\prime} \mathrm{W}$ & 190 \\
\hline Valença do PI & $06^{\circ} 24^{\prime} \mathrm{S}$ & $41^{\circ} 45^{\prime} \mathrm{W}$ & 295 \\
\hline Floriano & $06^{\circ} 46^{\prime} \mathrm{S}$ & $43^{\circ} 01^{\prime} \mathrm{W}$ & 85 \\
\hline Oeiras & $07^{\circ} 01^{\prime} \mathrm{S}$ & $42^{\circ} 08^{\prime} \mathrm{W}$ & 170 \\
\hline Picos & $07^{\circ} 05^{\prime} \mathrm{S}$ & $41^{\circ} 28^{\prime} \mathrm{W}$ & 195 \\
\hline São João do PI & $08^{\circ} 22^{\prime} \mathrm{S}$ & $42^{\circ} 15^{\prime} \mathrm{W}$ & 244 \\
\hline Canto do Buriti & $08^{\circ} 07^{\prime} \mathrm{S}$ & $42^{\circ} 57^{\prime} \mathrm{W}$ & 280 \\
\hline São Raimundo Nonato & $09^{\circ} 01^{\prime} \mathrm{S}$ & $42^{\circ} 41^{\prime} \mathrm{W}$ & 386 \\
\hline Bom Jesus & $09^{\circ} 04^{\prime} \mathrm{S}$ & $44^{\circ} 21^{\prime} \mathrm{W}$ & 220 \\
\hline Corrente & $09^{\circ} 04^{\prime} \mathrm{S}$ & $45^{\circ} 09^{\prime} \mathrm{W}$ & 434 \\
\hline Uruçuí & $07^{\circ} 14^{\prime} \mathrm{S}$ & $44^{\circ} 33^{\prime} \mathrm{W}$ & 167 \\
\hline
\end{tabular}

TABELA 2 - Valores de Tq, P, Im e os resultados do balanço hídrico, pelo método de Thornthwaite \& Mather (1955), admitindo-se um solo com capacidade de água disponível (CAD) igual a $100 \mathrm{~mm}$.

\begin{tabular}{lcccc}
\hline \multicolumn{1}{c}{ Municípios } & Tq $\left({ }^{\circ} \mathrm{C}\right)$ & P & Im & $\begin{array}{c}\text { B.H } \\
\text { ANUAL }\end{array}$ \\
& & & & \\
& & & & \\
\hline 1 - Parnaíba & 29,2 & $1.328,6$ & $-30,6$ & $-586,2$ \\
2 - Piripiri & 28,2 & 1584,2 & $-5,2$ & $-87,6$ \\
3 - Campo Maior & 28,7 & 1293,0 & $-26,6$ & $-469,0$ \\
4 - Teresina & 29,1 & 1377,7 & $-24,9$ & $-456,4$ \\
5 - São Pedro do PI & 27,8 & 1254,6 & $-16,2$ & $-288,4$ \\
6 - Valença do PI & 27,9 & 1070,4 & $-31,5$ & $-491,8$ \\
7 - Floriano & 29,5 & 950,6 & $-48,6$ & $-900,2$ \\
8 - Oeiras & 29,3 & 949,4 & $-48,1$ & $-879,9$ \\
9 - Picos & 29,1 & 706,7 & $-60,8$ & $-1095,9$ \\
10 - São João do PI & 29,3 & 712,7 & $-60,4$ & $-1085,7$ \\
11 - Canto do Buriti & 28,5 & 805,8 & $-50,7$ & $-830,2$ \\
12 - São Raimundo Nonato & 28,4 & 696,8 & $-56,4$ & $-902,8$ \\
13 - Bom Jesus & 28,6 & 951,3 & $-39,9$ & $-630,9$ \\
14 - Corrente & 27,3 & 1094,4 & $-20,1$ & $-275,9$ \\
15 - Uruçuí & 28,8 & 1103,2 & $-33,1$ & $-546,8$ \\
\hline
\end{tabular}

$\mathrm{Tq}=$ temperatura média do mês mais quente

$\mathrm{P}=$ precipitação média anual

B.H ANUAL = Balanço hídrico anual

Im = índice de umidade anual

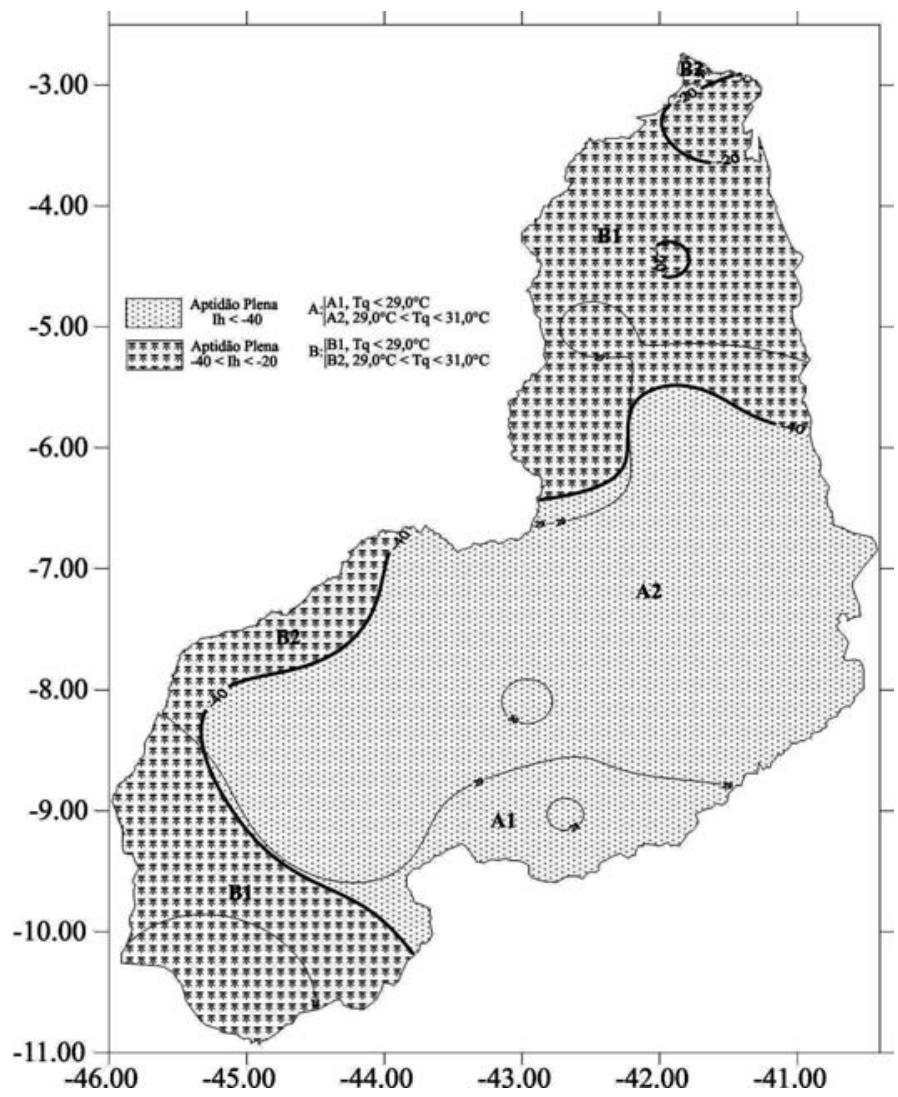

FIGURA 1- Aptidão climática para a cultura da mangueira no Estado do Piauí.

\section{CONCLUSÃO}

À luz dos critérios utilizados e com base na literatura consultada, o Estado do Piauí não apresenta nenhuma restrição climática para o cultivo comercial da mangueira.

\section{REFERÊNCIAS}

BRUNINI, O.; ALFONSI, R. R. Aptidão ecológica para a Mangueira. In: SIMPÓSIO BRASILEIRO SOBRE A CULTURA DA MANGUEIRA, 1., 1980, Jaboticabal. Anais... Jaboticabal: UNESP, 1980. p. $23-33$.

CAMPBELL, R. J. A guide to mangos in Flórida, Miami: Ed Fairchild Tropical Gardens, 1994. p.188-191.

CASTRO, P.R.C.; KLUGE, R. A. (Coord.). Ecofisiologia de fruteiras tropicais: abacaxizeiro, maracujazeiro, mangueira, bananeira e cacaueiro. São Paulo: Nobel, 1998.

FRANCISCO, V.L. F. dos S.; CASER, D. V.; AMARO, A. A. Perfil da cultura da manga em São Paulo. Informações Econômicas, São Paulo, v.33, n. 3, mar. 2003.

KRISHNAN, A. Agroclimatic classification methods and their application to Índia. Patancheru, Índia: ICRISAT, 1980. p. 59-88. 
LIMA, M. G.; RIBEIRO, V. G. Equação de estimativa da temperatura do ar para o Estado do Piauí. Revista Brasileira de Agrometeorologia, Santa Maria, n. 2, p. 221 -227, 1998.

MEDEIROS, A. A. do; AMORIM, J. R. A. de; SILVA; GUEDES, F. X.; GUERRA, A. G.; DANTAS, J. A. Composição mineral de folhas e exportação de nutrientes pelos frutos de magueira em cultivo irrigado no Rio Grande do Norte. Natal, RN: EMPARN, 2005.

PINHEIRO, J. M.; ASSIS, J. S. de; TEIXEIRA, A. H. de C.; CUNHA, G. A. P. de; NETP, M. T. de C. Ecofisiologia. In: GENU, P. J. de C.; PINTO, A. C. de Q. (Coord.). A cultura da manga. Brasília: Embrapa Informação Tecnológica, 2002. p.39-49.

SCHAFFER, B.; ANDERSEN, P. C. (Ed). Handbook of environmental physiology of fruit crops: sub- tropical and tropical crops. Flórida: CRC Press, 1994. v.2.
SENTELHAS, P.C.; PEREIRA, A. R.; MARIN, F. R.;ANGELOCCI, L. R.; ALFONSI, R.R.; CARAMORI, P.H.; SWART, S. Balanços hídricos climatológicos do Brasil. Piracicaba: ESALQ/USP, 1999. CD-ROM.

SILVA, W. J. Aptidão climática da cultura da mangueira. Informe Agropecuário, Belo Horizonte, v.8, n.86, p.5-8, 1982.

TEIXEIRA, A. H. de C.; AZEVEDO, P. V. Zoneamento agroclimático para a videira européia no Estado de Pernambuco, Brasil. Revista Brasileira de Agrometeorologia, Campinas, v.4, n.1, p.139-145, 1996.

THORNTWAITE, C.W.; MATHER, J.R. The water balance. New Jersey: Drexel Institute of Technology, 1955. 104 p. 